\title{
Is contact angle a cause or an effect? - A cautionary tale
}

\author{
Douglas Ruth
}

University of Manitoba, Winnipeg, Manitoba, Canada

\begin{abstract}
The most influential parameter on the behavior of two-component flow in porous media is "wettability". When wettability is being characterized, the most frequently used parameter is the "contact angle". When a fluid-drop is placed on a solid surface, in the presence of a second, surrounding fluid, the fluid-fluid surface contacts the solid-surface at an angle that is typically measured through the fluid-drop. If this angle is less than $90^{\circ}$, the fluid in the drop is said to "wet" the surface. If this angle is greater than $90^{\circ}$, the surrounding fluid is said to "wet" the surface. This definition is universally accepted and appears to be scientifically justifiable, at least for a static situation where the solid surface is horizontal. Recently, this concept has been extended to characterize wettability in non-static situations using high-resolution, two-dimensional digital images of multicomponent systems. Using simple thought experiments and published experimental results, many of them decades old, it will be demonstrated that contact angles are not primary parameters - their values depend on many other parameters. Using these arguments, it will be demonstrated that contact angles are not the cause of wettability behavior but the effect of wettability behavior and other parameters. The result of this is that the contact angle cannot be used as a primary indicator of wettability except in very restricted situations. Furthermore, it will be demonstrated that even for the simple case of a capillary interface in a vertical tube, attempting to use simply a two-dimensional image to determine the contact angle can result in a wide range of measured values. This observation is consistent with some published experimental results. It follows that contact angles measured in two-dimensions cannot be trusted to provide accurate values and these values should not be used to characterize the wettability of the system.
\end{abstract}

\section{Introduction}

This paper will discuss various considerations of the interface between a gas, a liquid, and a solid surface. The fundamental principle of a force balance between the gas and the liquid, if they are separated by a curved surface, is that the difference in pressure across that surface, $\Delta P$, is given by

$$
\Delta P=\sigma_{l g}\left(\frac{1}{R_{1}}+\frac{1}{R_{2}}\right)
$$

Here $\sigma_{l g}$ is the interfacial tension between the gas and the liquid, and $R_{1}$ and $R_{2}$ are the two radii of curvature of the surface. If a drop of fluid is placed on a flat, solid surface, a balance of forces requires that (Young's equation)

$$
\sigma_{l g} \cos \theta=\sigma_{s g}-\sigma_{s l}
$$

Here $\sigma_{s g}$ is the interfacial tension between the solid and the gas, $\sigma_{s l}$ is the interfacial tension between the solid and the liquid, and $\theta$ is the angle that the liquid-gas surface makes with the solid surface, termed the "contact angle". If the solid is a circular capillary tube, then the pressure difference across the surface is termed "capillary pressure", $P_{c}$, and simple rules of geometry lead to the expression

$$
P_{c}=\frac{4 \sigma_{l g} \cos \theta}{\delta}
$$

Here $\delta$ is the diameter of the tube. These three equations are all that is required to demonstrate the characteristic behaviors of contact angles.

Generally, Equation 2 is used to determine the wettability of a three component (liquid-gas-solid) system. Specifically, if $\theta$ when measured through the liquid is found to be much less than $90^{\circ}$, the system is said to be strongly "liquid wet"; a typical liquid wet system is water/ air/glass. If $\theta$ is near $90^{\circ}$ the system is said to be "neutrally wet". If $\theta$ is much greater than $90^{\circ}$, the system is said to be "gas wet"; a typical gas wet system is mercury/ air/ glass. For very simple cases, such as a drop of water on a horizontal solid surface, this definition of wettability is universally accepted. Unfortunately, this definition has become dogmatic and it has been extended to mean that the contact angle directly defines the wettability in all situations. In particular, with the advent of high resolution imaging methods, apparent contact angles can be measured insitu in both static and dynamic situations. In the present paper, published results and simple thought experiments are used to demonstrate that contact angles are not always characteristic of wettability. Furthermore, it is demonstrated that direct measurement of in situ contact angles is difficult without sophisticated interpretation methods.

This paper is not a condemnation of the ability to determine contact angles on the microscopic scale - it is a cautionary statement that such determinations must be done with great care. However, this paper does demonstrate that interpreting wettability from direct contact angle measurements is not always a meaningful activity.

\section{The Contact Angle and Wettability}

It is a tacit assumption that if a contact angle can be measured, then the wettability of a system can be determined. However, there are many cases where this assumption is obviously wrong. One of these cases is demonstrated in the classic monograph by Craig [1]. His Fig. 2.3 clearly demonstrates that even in the case of a static contact angle measurement, it can take many 
hundreds of hours to obtain an equilibrium contact angle. The illustration is for an oil/ water/ solid surface and it could be argued that such systems are susceptible to complex chemical factors. However, even this simple case provides a cautionary note. Modelling of displacement processes generally utilize capillary pressure data that is time-independent. But if the contact angle is time dependent, then it follows from Equation 3 that the capillary pressure must be time dependent. Therefore, all displacement processes are potentially time dependent, an issue rarely considered when analyzing such processes.

A second case of interest is that of a drop on a surface, when the surface is gradually tilted from the horizontal. This case has been studied since at least the late 1930s [2]. A more recent paper by Krasovitski and Marmur [3] provides a succinct description of this case. What they found is that the drop distorts but does not move until the plate is tilted to a critical angle, the "slip angle". Assuming, a gas/ water/ solid system, when the drop distorts, the contact angle at the up-tilt end of the drop, $\theta_{u}$, decreases and the contact angle at the downtilt end of the sample, $\theta_{d}$, increases. Using the conventional interpretation, this would mean that simply tilting the surface causes the up-tilt end of the system to become more water-wet while the down-tilt end of the system moves toward neutral-wettability

It is found both experimentally and theoretically that the slip angle, $\alpha$, is related to the two contact angles by the relation

$$
\sin \alpha=C \sigma_{l g}\left(\cos \theta_{u}-\cos \theta_{d}\right)
$$

Here $C$ is a constant that depends on the densities of the fluids, gravitational acceleration, and drop shape. Krasovitski and Marmur also document that once the drop begins to move, the values of $\theta_{u}$ and $\theta_{d}$ do not remain constant. An argument can be made that these changes are due to the fact that the up-tilt contact line is receding over a surface which was in contact with water, while the down-tilt contact line is advancing over a surface that was in contact with gas, and that this difference accounts for the difference between "advancing contact angles" and "receding contact angles". However, at tilt angles less than the slip angle, this argument does not apply because the drop has not yet moved.

Next, consider a water/ air/ solid system in a vertical capillary tube with one end immersed in a tray of liquid and the other open to air. The well-known behavior is that water will rise in the tube until a force balance obtains between the hydrostatic and capillary forces. The pressure in the water at the water-surface in the tray must be equal to the pressure in the air at this point. Therefore, this force balance requires that

$$
\frac{4 \sigma_{l g} \cos \theta}{\delta}=\left(\rho_{l}-\rho_{g}\right) g h
$$

Here $\rho_{l}-\rho_{g}$ is the difference between the densities of water and air, $g$ is the acceleration of gravity, and $h$ is the height of the liquid/ air interface in the capillary tube relative to the height of the liquid/ gas interface in the tray.

Now consider what would happen if the tube is gently removed from the tray and a second water/ air surface is created at the bottom of the tube. The pressure below the bottom water/ air surface differs from the pressure above the top water/ air surface by $\rho_{g} g h$. Therefore, the hydrostatic pressure of the water in the tube just makes up for the capillary pressure at the upper surface and the pressure difference at the bottom water/ air surface must remain at zero. But this implies that the bottom surface is flat and that $\theta$ is $90^{\circ}$. Using our conventional definition of wettability means that the top surface is strongly water-wet and the bottom surface is neutrally-wet.

Continuing this line of reasoning, various amounts of water can be forced from the tube to obtain force balances that would require

$$
\begin{gathered}
\frac{4 \sigma_{l g}}{\delta}\left(\cos \theta_{\text {top }}-\cos \theta_{\text {bottom }}\right)= \\
\left(\rho_{l}-\rho_{g}\right) g h
\end{gathered}
$$

Therefore, by implication, the contact angle at the bottom water/air surface would have values given by the equation

$$
\begin{aligned}
& \theta_{\text {bottom }}= \\
& \operatorname{arcos}\left(\cos \theta_{\text {top }}-\frac{\delta\left(\rho_{l}-\rho_{g}\right) g h}{4 \sigma_{l g}}\right)
\end{aligned}
$$

This equation implies that wettability at the bottom of the tube varies continuously with height of water.

The above description is an approximation to what would actually happen experimentally. The bottom of the tube includes a flat surface which complicates the situation. Water can spread across this surface resulting is a convex-up surface that increases the pressure at the bottom surface, hence the pressure at the top surface. However, the conclusion remains the same - the contact angle at the top surface cannot be the same as the contact angle at the bottom surface or the tube will drain and experimentally it is found that the tube does not drain.

A variation on the above reasoning can be made for a horizontal tube that is spun in a centrifuge. The equation describing the capillary pressure in this system is

$$
P_{c o}-P_{c i}=\left(\rho_{g}-\rho_{l}\right) \frac{\omega^{2}}{2}\left(r_{o}^{2}-r_{i}^{2}\right)
$$

Here $r$ is the radius of rotation, the subscript $i$ denotes the variables at the fluid interface closest to the center of rotation, $o$ denotes the variables at the interface farthest from the center of rotation, and $\omega$ is the angular velocity. At zero angular velocity, the pressures in the water at the two ends of the tube are the same. As the angular velocity is increased, the equilibrium condition requires that the capillary pressures at the two ends of the tube must differ. Using Equation 3, this difference can be expressed in terms of contact angles: 


$$
\begin{aligned}
& \frac{4 \sigma_{l g}}{\delta}\left(\cos \theta_{o}-\cos \theta_{i}\right)= \\
& \quad\left(\rho_{g}-\rho_{l}\right) \frac{\omega^{2}}{2}\left(r_{o}{ }^{2}-r_{i}{ }^{2}\right)
\end{aligned}
$$

We can conclude from this that the contact angle for the interface farthest from the center of rotation varies with angular velocity; hence, following conventional reasoning, the wettability at this point, is a function of angular velocity which is not true.

There are also many reports in the literature of how the contact angle at the interface of two fluids in a tube differ with the velocity of the interface. It is well known that the problem of a moving contact angle cannot be solved analytically because a singularity arises in the equations at the contact point of the two fluids and the solid surface. This singularity is generally treated by assuming that there is "slip" at the contact line. Based on this model, the classic paper by Cox [4] showed that the apparent contact angle was related to the static contact angle as a function of the capillary number. This behavior was verified experimentally in such works as Fermigier and Jenffer [5]. It is found that, above a capillary number of approximately $10^{-3}$, the apparent contact angle can vary significantly, going from static values of $30^{\circ}$ to values approaching $180^{\circ}$. In terms of wettability, these contact angles imply a variation from strongly water wet to strongly oil wet. Therefore, for dynamic displacements in capillary tubes, the contact angle cannot be used to characterize the wettability. If this is true for flow in capillary tubes, it must also be true for flow in porous media.

\section{In-Situ Measurements of Contact Angles}

There has been a recent interest in measuring contact angles in-situ by means of micro-imaging techniques (e.g. Andrew et al [5], Held et al [6]). However, similar to the measurement of porosity by means of twodimensional images, in-situ measurement of contact

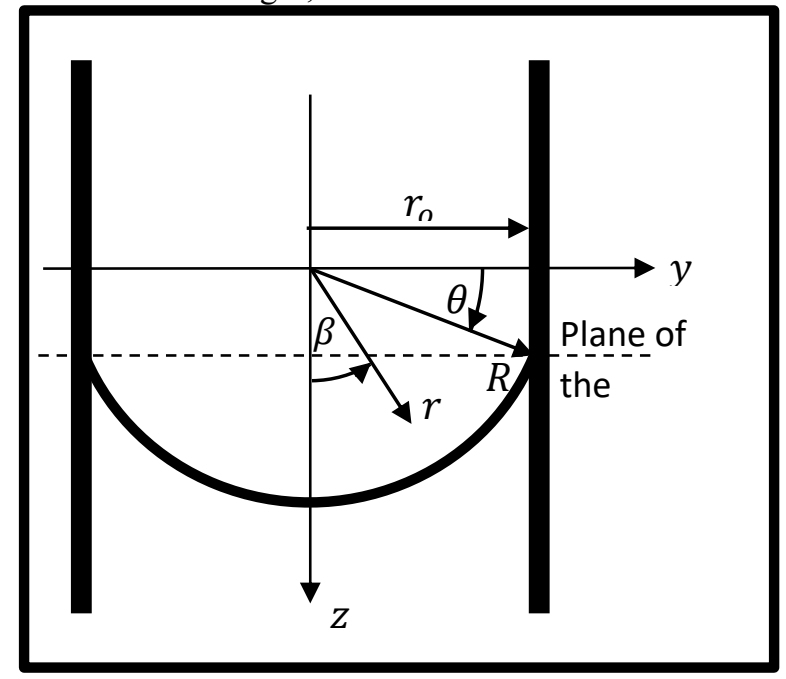

Fig. 1a Nomenclature for an interface in a vertical tube (elevation view). angles are problematic. This will be illustrated by using the case of an interface in a vertical capillary tube.

Fig. 1 shows the nomenclature for an interface in a vertical tube. The interface is assumed to have a spherical shape with a radius of curvature, $R$. Two coordinate systems will be used: a Cartesian system with coordinates $x, y, z$ and a spherical coordinate system with coordinates $r, \alpha, \beta$. The origin of both coordinate systems is the center of curvature of the surface. Here, $Z$ is assumed positive downward.

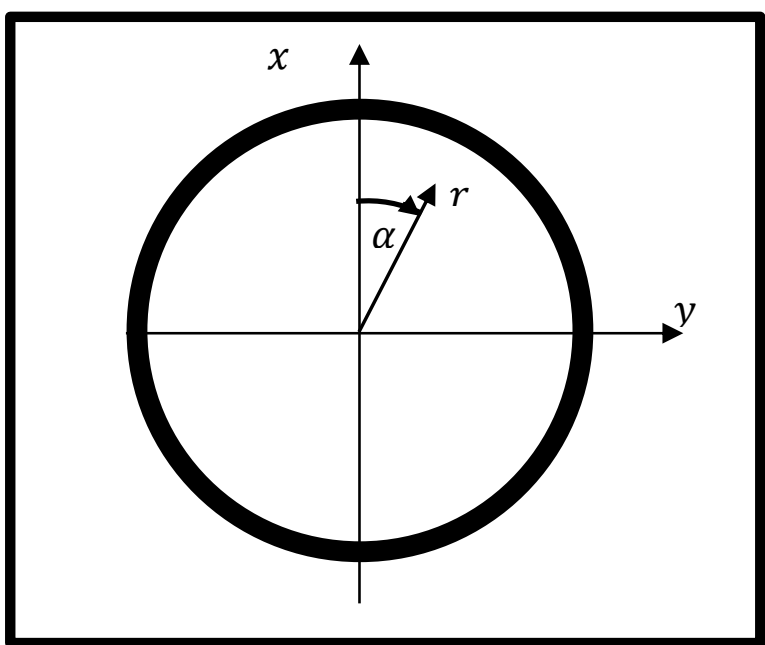

Fig. 1b Nomenclature for an interface in a vertical tube (plan view).

The equations of the surface in the Cartesian coordinate system in terms of the spherical system are

$$
\begin{gathered}
x_{s}=R \sin \beta \cos \alpha \\
y_{s}=R \sin \beta \sin \alpha \\
z_{s}=R \cos \beta
\end{gathered}
$$

The equations for the wall in Cartesian coordinates in terms of the spherical system are

$$
\begin{gathered}
x_{w}=R \sin \theta \cos \alpha \\
y_{w}=R \sin \theta \sin \alpha \\
z_{w}=z
\end{gathered}
$$

It is next assumed that the interface is cut by a plane, as shown in Fig. 2, such that the cross-section of the interface can be examined in a two-dimensions plane, $u-w$. (This plane is meant to represent the observation plane in a two-dimensional imaging experiment.) The equations for this plane in terms of the Cartesian coordinate system are

$$
\begin{gathered}
x_{p}=u \\
y_{p}=y_{e}-w \cos \gamma \\
z_{p}=z_{e}+w \sin \gamma
\end{gathered}
$$


The equations for the surface in the twodimensional plane in terms of the spherical system can be found by combining Equations 9 and 11. The result is

$$
\begin{gathered}
u=R \sin \beta \cos \alpha \\
w=\frac{R \cos \beta-z_{e}}{\sin \gamma} \\
w=\frac{y_{e}-R \sin \beta \sin \alpha}{\cos \gamma}
\end{gathered}
$$

The two equations for $w$ require that

$$
\begin{aligned}
& \left(\cos \beta-z^{\prime}{ }_{e}\right) \cos \gamma= \\
& \quad\left(y_{e}^{\prime}-\sin \beta \sin \alpha\right) \sin \gamma
\end{aligned}
$$

where $y^{\prime}{ }_{e}$ is $y_{e} / R$ and $z^{\prime}{ }_{e}$ is $z_{e} / R$.

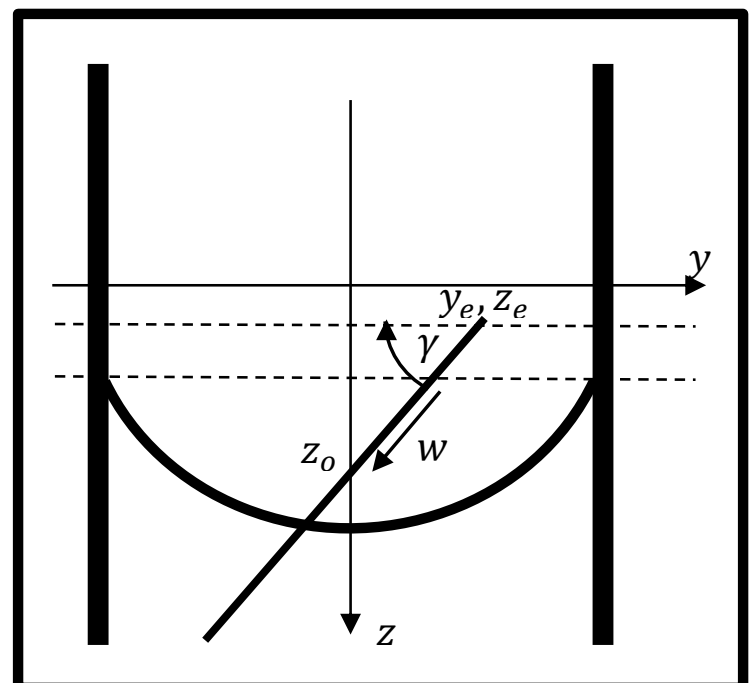

Fig. 2 A two dimensional plane that intersects the surface and the wall.

The two-dimensional surface can be characterized by the point where the plane crosses the $z$ - axis, $z_{o}$, and the angle the plane makes with the horizontal, $\gamma$. This will allow the calculation of the coordinates where the plane crosses the surface in the cross-section shown in Fig. 2. Provided that the plane intersects the plane of contact, the minimum $\beta$-value, denoted by $\beta_{1}$, will always be $\pi / 2-\theta$ (the point where the surface contacts the wall). For this condition,

$$
\begin{gathered}
w=\frac{R \sin \theta-z_{e}}{\sin \gamma}=\frac{y_{e}-R \cos \theta}{\cos \gamma} \\
\tan \gamma>\frac{z_{o}}{r_{o}}
\end{gathered}
$$

If the intersecting plane does not intersect the plane of contact, then the $\beta$-value can be found using Equation 13 and setting the $\alpha$-values to $\pi / 2$; this $\beta_{1}$-value is given by

$$
\begin{gathered}
\sin \beta_{1}=\left(z^{\prime}{ }_{e} \cos \gamma+y^{\prime}{ }_{e} \sin \gamma\right) \sin \gamma- \\
\cos \gamma \sqrt{\left(1-\left(z^{\prime}{ }_{e} \cos \gamma+y^{\prime}{ }_{e} \sin \gamma\right)^{2}\right)}
\end{gathered}
$$

The second point where the plane intersects the surface, $\beta_{2}$, is also given by Equation 13. Again setting the $\alpha$ values to $\pi / 2$, this time the solution is

$$
\begin{aligned}
& \sin \beta_{2}=\left(z^{\prime}{ }_{e} \cos \gamma+y^{\prime}{ }_{e} \sin \gamma\right) \sin \gamma+ \\
& \cos \gamma \sqrt{\left(1-\left(z^{\prime}{ }_{e} \cos \gamma+y^{\prime}{ }_{e} \sin \gamma\right)^{2}\right)}
\end{aligned}
$$

Furthermore, Equation 13 can be used to generate $\alpha$ values that correspond to $\beta$-values

$$
\begin{aligned}
& \alpha= \\
& \sin ^{-1}\left(\frac{y^{\prime}{ }_{e} \sin \gamma+z^{\prime}{ }_{e} \cos \gamma-\cos \beta \cos \gamma}{\sin \beta \sin \gamma}\right)
\end{aligned}
$$

The shape of the surface in the intersecting plane can therefore be calculated for various $\beta$-value between the minimum and the maximum.

For the wall, the coordinates in the intersecting plane become

$$
\begin{gathered}
u=R \cos \theta \cos \alpha \\
w=\frac{y_{e}-R \cos \theta \sin \alpha}{\cos \gamma}
\end{gathered}
$$

The shape of the wall in the intersecting plane can therefore be calculated for any $\alpha$-value. For the wall, $\alpha$-values range from 0 to $2 \pi$.

A special case is when the intersecting plane is vertical. Then the equation for the plane is

$$
\begin{gathered}
x=R \cos \beta \cos \alpha \\
y=y_{o} \\
z=z_{p}
\end{gathered}
$$

the intersection of the surface in the plane is

$$
R \cos \beta \sin \alpha=y_{o}
$$

$$
y_{p}=R \sin \beta
$$

the maximum $\beta$-value is

$$
\beta=\cos ^{-1}\left(\frac{y_{o}}{R}\right)
$$

and the $\alpha$-values are given by

$$
\alpha=\sin ^{-1}\left(\frac{y_{o}}{R \cos \beta}\right)
$$

The apparent contact angle can be found from

$$
\left(\frac{d w}{d u}\right)_{\text {surface }}-\left(\frac{d w}{d u}\right)_{\text {wall }}=\tan \theta_{a}
$$

evaluated at the wall. This is a complex derivative best done numerically.

\section{Typical Interface Shapes}

Fig. 3 through 9 show typical interface shapes for planes that intersect the interface at various positions. These figures were calculated assuming that $\theta=30^{\circ}$. In each figure, the left panel shows a schematic of the intersection and the right panel shows what would be observed in the intersecting plane, that is, the plane of observation in an imaging experiment.

Fig. 3 shows the case where the plane intersects the interface horizonally. The conclusion that could be drawn from the image is that gas is flowing as an encapsulated phase and that the water is perfectly wetting with an apparent contact angle of $\theta_{a}=0$. In 
this case, the intersection of the plane with the wall is circular.

Fig. 4 shows the case where the plane intersects the interface before it intersects the wall. The conclusion that could be drawn from the image is that gas is flowing as an encapsulated phase, that the water is perfectly wetting with an apparent contact angle of $\theta_{a}=0$, and perhaps water is trapped in a pore space located at the bottom of the image. In this case, the intersections of the plane with the wall is elliptical and the plane with the interface is circular.

Fig. 5 shows the case where the plane intersects the surface at the point where the interface contacts the wall. In this case, $\gamma \cong 20^{\circ}$ and the apparent contact angle that would be measured in the image is $\theta_{a}=0$. In this case, the intersections of the plane with the wall is elliptical and the plane with the interface is circular.

Fig. 6 shows the case where the plane intersects the surface at the point such that $=45^{\circ}$; the apparent contact angle that would be measured in the image is $\theta_{a}=24.5^{\circ}$. In this case, the intersection of the plane with the wall is elliptical.

Fig. 7 shows the limiting case where the plane is vertical, $\gamma=90^{\circ}$; the apparent contact angle that would be measured is the true contact angle $\theta_{a}=30^{\circ}$. In this case, the intersection of the plane with the wall is elliptical; however, the ellipse is infinitely extended such that the wall appears as two vertical lines.

Fig. 8 shows the case where the plane is vertical, $\gamma=90^{\circ}$, but is offset from the $z$-axis half the distance to the wall. In this case the apparent contact angle that would be measured is $\theta_{a}=33.7^{\circ}$. The wall still appears as two vertical lines; however, the spacing between the lines is less than for the previous case. This could be interpreted to mean that the image is for a capillary tube with a smaller diameter than in the previous case.

Fig. 9 shows the case where the plane is vertical, $\gamma=90^{\circ}$, but is offset from the $z$-axis by $95 \%$ of the distance to the wall. In this case the apparent contact angle that would be measured is $\theta_{a}=61.6^{\circ}$. The wall still appears as two vertical lines; however, the spacing between the lines is much less than for the previous case. This could be interpreted to mean that the image is for a capillary tube with a much smaller diameter than in the previous case.

The implications of the last two cases is obvious. Simply examining the images to obtain apparent contact angles, it could be concluded that the contact angles for smaller sized capillary tubes increase and approach $\theta_{a}=90^{\circ}$ in the limit. This would suggest that smaller capillary tubes are neutrally wet while larger tubes are water wet. This conclusion is, of course, specious.
In summary, using intersecting planes for various positions, it is possible to measure apparent contact angles anywhere in the range $0^{\circ} \leq \theta_{a} \leq 90^{\circ}$. This conclusion is completely supported by the work of Andrew et al [6]. They used what they called a "resampling plane" that could be reoriented relative to the grain surface. They found that the measured angle could vary from approximately $10^{\circ}$ to $110^{\circ}$ (their Fig. 10) by repositioning the resampling plane.

The above analysis considers the very simple example of a straight, circular capillary tube. Real porous media have flow passages that diverge, converge, twist and turn. An analysis of such passages is beyond the scope of the present paper. However, it is obvious that the measurement of true contact angles in such passages would be much more complex than that for the simple model considered here.

\section{A Way Forward}

It should be no surprise that the contact angle must be an effect, not a cause. Examining Young's equation, there are four parameters $\sigma_{l g}, \theta, \sigma_{s g}$, and $\sigma_{s l}$. Basic principles of functions requires that only three of these variables can be independent. The interfacial tensions are assumed to be properties of the substances, related to how various species of molecules interact across a surface. They can therefore be assumed to be the independent variables. This implies that the contact angle is the dependent variable and

$$
\theta=f\left(\sigma_{l g}, \sigma_{s g}, \sigma_{s l}\right)
$$

Furthermore, Young's equation depends on a static force balance and other force terms must affect that balance if they are present. It is unreasonable to expect the values of the interfacial tensions to change with the additional forces; hence, the contact angle must change. In fact, many theoretical studies utilize a modified Young's equation that includes body forces and fluid stresses.

I emphasize that this paper is not a condemnation of the ability to determine contact angles on the microscopic scale - it is a cautionary statement that such determinations must be done with great care. The work of Andrew et al [6] shows how the measured contact angle varies depending on how the plane of investigation is oriented. Their work could be taken one-step further. Using multiple images, a threedimensional interface model could be reconstructed and this model could then be interpreted to determine a unique contact angle that characterizes the system, assuming that such an angle exists. Based on observations made in the Andrew et al work, such a process would require much more detailed images than they acquired. They found that there was insufficient accuracy in the digital data to determine contact angles computationally and they resorted to a manual process. This would be impractical if reconstructions of interfacial surfaces are performed, particularly in a dynamic experiment. It would appear that one more 
level of sophistication in image analysis must be achieved before contact angles can be systematically interpreted from micro-imaging experiments. There is evidence that this type of work is being pursued (AlRatrout et al [8]).

\section{Conclusions}

The present work supports the following conclusions:

1. Contact angles are dependent variables, functions of fluid/ solid properties (interfacial tensions), displacement velocities, history (time, hysteresis, and externally applied body forces and viscous stresses) - they are effects not causes.

2. Wettability can be defined dependably by the contact angle only for the static case with no externally imposed forces. Contact angles measured in dynamic and complex systems may not be good indictors of wettability.

3. It is not possible to measure accurately a contact angle in a simple two-dimensional image. Such measurements would require detailed reconstruction and interpretation of the threedimensional interface.

\section{References}

1. F.F.Craig, jr., The Reservoir Engineering Aspects of Waterflooding, (SPE Monograph Series, 3, 1971).

2. G.Macdougall, and C.Ockrent, "Surface energy relations in liquid/solid systems. I. The adhesion of liquids to solids and a new method of determining the surface tension of liquids", PRSA 180, 981, 151-173, (1942).

3. B.Krasovitski and A.Marmur,'Drops down the hill: Theoretical study of limiting contact angles and the hysteresis range on a tilted plate", Langmuir, 21, 3881-3885, (2005).

4. R.G.Cox, "The dynamics of spreading of liquids on a solid surface. Part 1. Viscous flow”, JFM, 168. 169-194, (1986).

5. M.Fermigier and P.Jenffer, "An experimental investigation of the dynamic contact angle in liquidliquid systems", JCIS, 146, 1, 226-241, (1991).

6. M.Andrew, B.Bijeljic, and M.J.Blunt, "Pore-scale contact angle measurements at reservoir conditions using X-ray microscopy”, AWR, 68, June, 24-31, (2014).

7. R.Held, N.Schleifer, L.Genolet, and A.Fogden, "Added insight from image-based wettability characterization", E3S Web Conference, 89, (International Symposium of the Society of Core Analysts, 2018).

8. A.AlRatrout, A.Q.Raeini, B.Bijeljic, and M.Blunt, "Automatic measurement of contact angle in porescale images", AWR, 109, 158-269, (2017). 

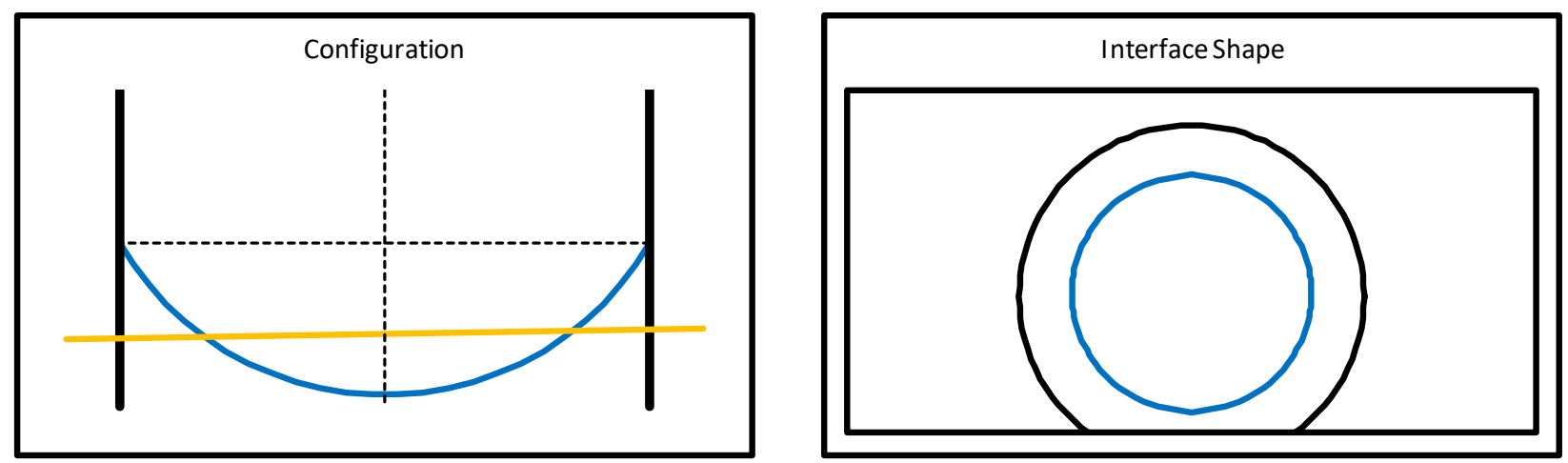

Fig. 3 The shape of the interface in a horizontal plane that cuts the interface. The water lies below the blue line and the air above.
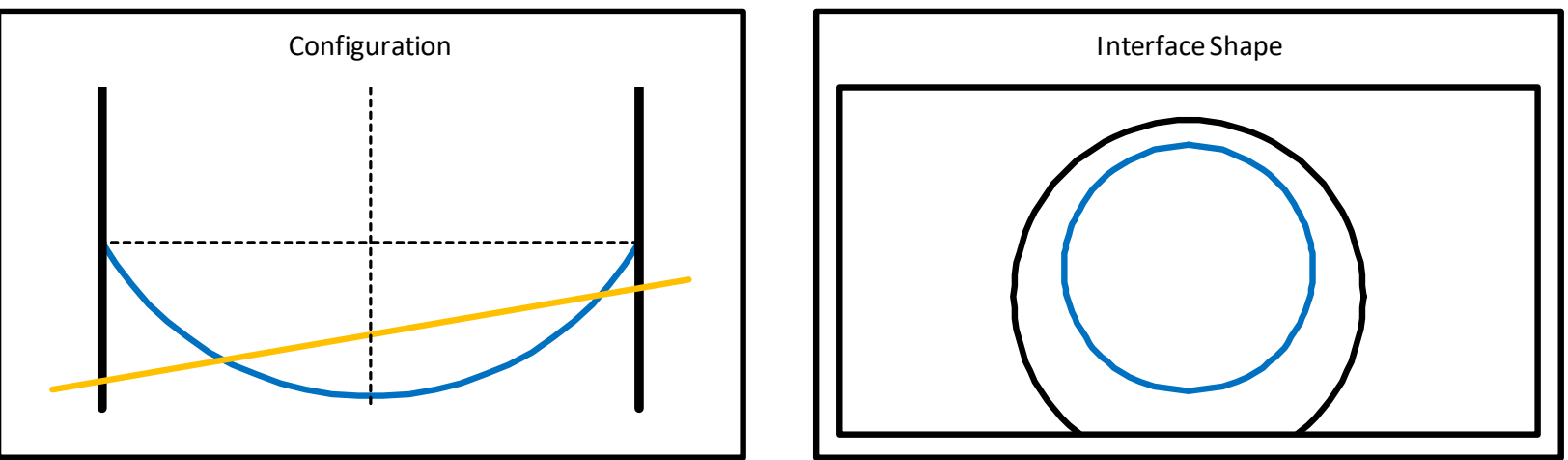

Fig. 4 The shape of the interface in a plane that crosses the interface before it crosses the wall. The water lies below the blue line and the air above.
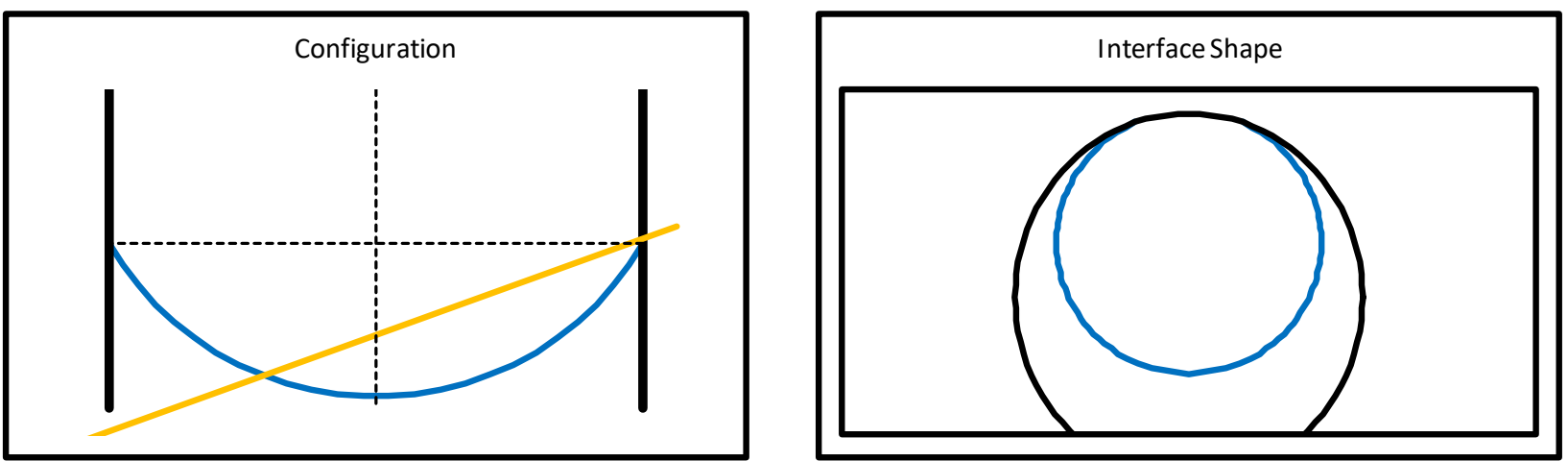

Fig. 5 The shape of the interface in a plane that intersects the interface at the wall. The water lies below the blue line and the air above.
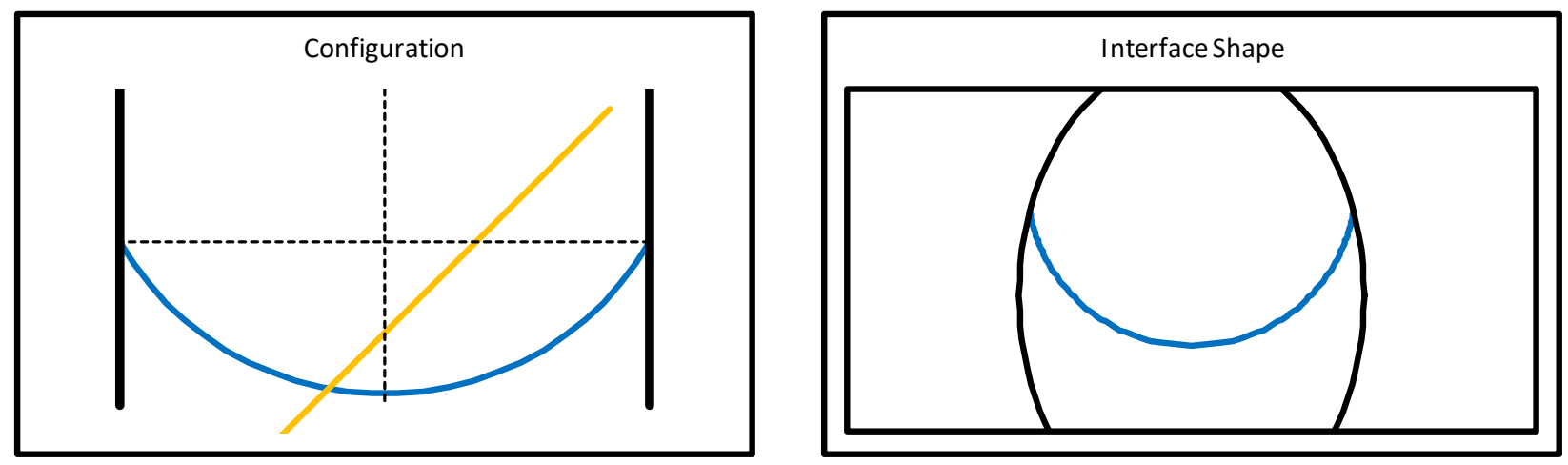

Fig. 6 The shape of the interface in a plane for $\gamma=45^{\circ}$

The water lies below the blue line and the air above. 

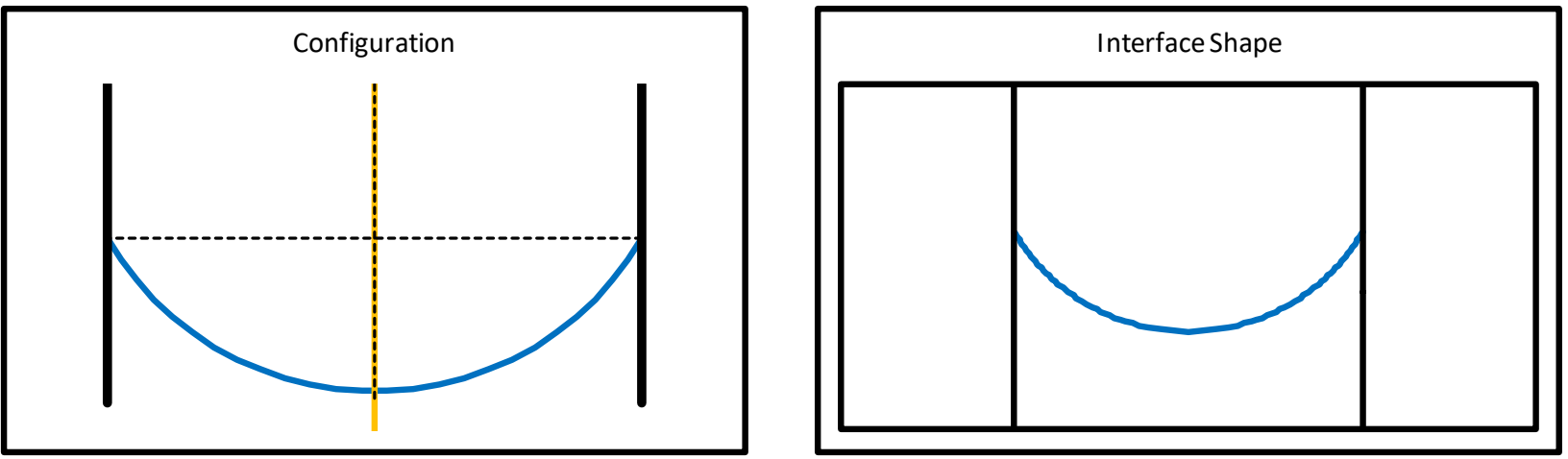

Fig. 7 The shape of the interface in a vertical plane. The water lies below the blue line and the air above.
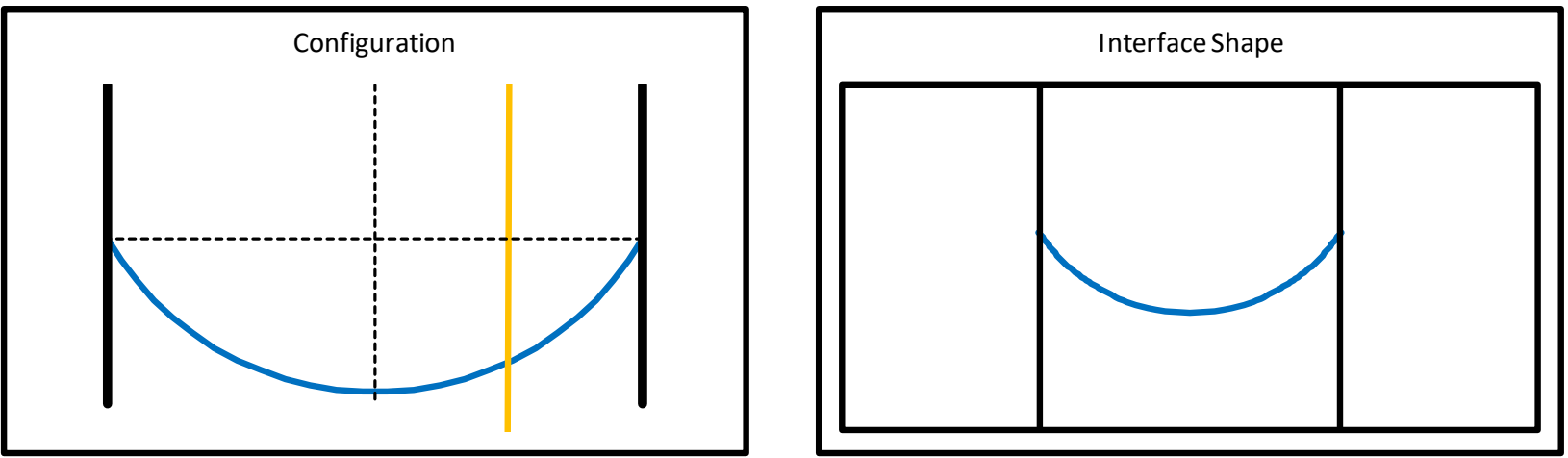

Fig. 8 The shape of the interface in a vertical plane offset from the $z$-axis half the distance to the wall. The water lies below the blue line and the air above.
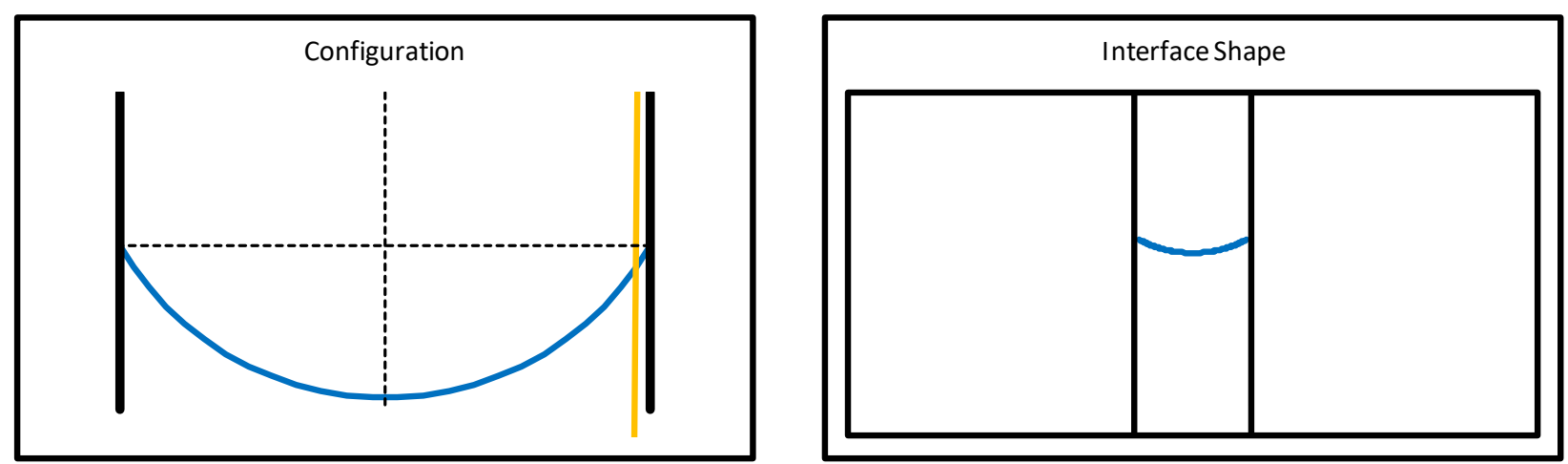

Fig. 9 The shape of the interface in a vertical plane offset from the $z$-axis is $95 \%$ of the distance to the wall. The water lies below the blue line and the air above. 